Collection SFN 10 (2010) 487-503

(C) Owned by the authors, published by EDP Sciences, 2010

DOI: $10.1051 / \mathrm{sfn} / 2010010$

\title{
High resolution inelastic X-ray scattering from thermal collective excitations
}

\author{
M. d'Astuto ${ }^{1}$ and M. Krisch² \\ 1 Institut de Minéralogie et de Physique des Milieux Condensés (IMPMC), CNRS UMR 7590, \\ Université Pierre et Marie Curie - Paris 6, case 115, 4 place Jussieu, 75252 Paris Cedex 05, \\ France \\ 2 European Synchrotron Radiation Facility, BP. 220, 38043 Grenoble Cedex, France
}

\begin{abstract}
In this lecture we will introduce the general characteristics of non-resonant X-ray inelastic scattering, in particular its scattering and absorption cross-section as well as its kinematic conditions. We will then present how it is possible to achieve meV energy resolution with $\mathrm{keV} \mathrm{X}$-rays, hence performing light inelastic scattering of thermal excitations with a wavelength of the order of inter-atomic spacing in liquids and solids. We will describe the perfect crystal monochromator back-scattering experimental set-up and the resulting instrumental function in energy and momentum space. We will then discuss the consequence of the above mentioned experimental characteristics, in particular for the application of the technique to phonon dispersion measurements in crystals, in comparison with triple axes inelastic neutron scattering and Raman experiments.
\end{abstract}

\section{INTRODUCTION}

To study the dispersion of thermal excitations in condensed matter, the probe needs to have a wavelength of the order of the inter-atomic distance and an energy resolution in the "thermal" range, i.e. $\sim 1-$ $1000 \mathrm{~K}$, or $\sim 0.1-100 \mathrm{meV}$. It is relatively simple to achieve this energy resolution using a probe having itself an energy of the same order. This is the case for photons in the infrared spectral region, but their wavelength is in the $0.1-1 \mathrm{~mm}$ range. Only neutrons fullfil both the energy and wavelength conditions : thermal neutrons have a wavelength of the order $\sim 1 \AA$ for an energy in the $10 \mathrm{meV}$ range, as seen in the previous chapters of this course. For photons, it is therefore necessary to use X-rays, which have a wavelength of the correct order (0.1-10 $\AA$ ). These photons, however, have an energy $10^{5}-10^{7}$ times higher than the thermal excitations. As a consequence, an extreme resolution of $\frac{\Delta E}{E} \sim 10^{-7}$ is required.

Inelastic scattering of X-rays by atomic vibrations is known since the first half of the $X X$ th century (see Ref. [1], chapter 5, p. 123 and references therein) as "thermal diffuse scattering" [2]. Such type of experiment is not resolved in energy, and it consists of measuring the diffuse scattering, between Bragg reflections, at different temperatures. It is then possible to separate the static diffuse scattering - originating from structural disorder and which is independent from temperature-, from the thermal disorder originating from phonons. The scattering cross section is relatively strong, roughly comparable to that of the neutron, as we will see in Section 2.

Spectrometers for inelastic X-ray scattering are of the triple axis type. They were developed by DuMond and Kirkpatrick [3,4] as early as 1930, using the Rowland geometry [5,6]. However, the relative energy resolution of $10^{-7}$ was only achieved recently, starting from the pioneering idea of Dorner and co-workers [7-9] and then pushed to the sub-THz range by Sette and co-workers [10-13].

This is an Open Access article distributed under the terms of the Creative Commons Attribution-Noncommercial License 3.0, which permits unrestricted use, distribution, and reproduction in any noncommercial medium, provided the original work is properly cited. 
This required the use of perfect single crystal optics as well as synchrotron X-ray sources as we will see in Section 3. The last section (section 4) of this lecture will give some examples to illustrate the characteristics of the technique. The results will be presented such as to underline the difference and complementarity between IXS, INS (triple axis spectrometers) and visible light spectroscopy (Raman).

\section{NON-RESONANT X-RAY INELASTIC SCATTERING}

\subsection{X-ray scattering and absorption cross section}

Photons with $\sim \AA$ wavelength, called X-rays, have energies in the range of $1-100 \mathrm{keV}$. In this range they can excite transitions between atomic "core" electron levels and valence or continuum states. In this situation, both absorption and scattering, called resonant in that case, have a peculiar behavior, strongly dependent on the energy fine structure of the levels involved in the transition [14]. These phenomena concern relatively narrow energy windows. Away from them, both absorption and scattering are simply proportional to the electron density, and have a smooth, decreasing energy dependence. We speak in this case of "non-resonant" scattering. For technical reasons, detailed below in Sec. 3, the experimental instrument we are going to describe is limited to specific energies far away from any of such atomic transition energies. Therefore, we can restrict ourselves to the case of non-resonant scattering and absorption processes, for which a detailed description is found in textbooks Ref. [14,15]. Here we would like to stress only one point which is important for the comparison with inelastic neutron scattering (INS) experiments: the non-resonant X-ray magnetic cross section is extremely weak [14, 16, 17]. It is possible, using some special experimental set-up, to measure non-resonant magnetic elastic scattering $[16,17]$, but inelastic processes yield a far too weak signal ${ }^{1}$.

Let us focus on the scattering cross section relevant for the experiment we are going to describe. The main contribution to the non-resonant X-ray scattering process is known as Thomson scattering, and is widely described in many reference texts [14,19], lectures [15] (Ch. 6) and reviews [20]. The mathematical formalism used to derive the expression of IXS cross section is very similar to that used to obtain the coherent nuclear INS cross section (see for example Ref. [1], chapter 5). Therefore the reader can refer to the previous chapter by $\mathrm{H}$. Schober in this book for details on the mathematics derivation of the expression. Here we will give only a brief description of its main characteristics in order to outline the differences with the nuclear neutron scattering cross section.

The double differential cross section can be written as :

$$
\frac{\partial^{2} \sigma}{\partial \Omega \partial E}=r_{0}^{2}\left(\hat{\epsilon}_{i} \cdot \hat{\epsilon}_{f}\right)^{2} \frac{k_{i}}{k_{f}} \sum_{I, F} P_{I}\left|\left\langle F\left|\sum_{j} e^{i \vec{Q} \cdot \vec{r}_{j}}\right| I\right\rangle\right|^{2} \delta\left(E-E_{f}-E_{i}\right)
$$

where $r_{0}=e^{2} / m_{e} c^{2}$ is the classical electron radius, $\hat{\epsilon}_{i(f)}$ is the photon incoming (out-coming) polarisation, $k_{i(f)}$ the norm of the photon wavevector, $P_{i}$ the thermal population of the initial quantum state of the material, $I(F)$ the initial (final) state of the system, $\vec{Q}=\vec{k}_{i}-\vec{k}_{f}$ the exchanged wavevector, $\vec{r}_{j}$ the position of the $j$ th electron in the system and $E_{i(f)}$ the energy of the photon before (after) the scattering process.

Assuming the adiabatic approximation is valid and with the restriction to the case where the electronic wave function is not modified by the scattering process -so that we consider only atomic

\footnotetext{
${ }^{1}$ Note that resonant X-ray scattering can have very strong magnetic contributions [14], and inelastic experiments are, in principle, feasible. The subject lies outside the purpose of the present work, as the instrument used does not allow such a high energy resolution $(\mathrm{meV})$ required for collective atomic excitations. Moreover, such experiments are very recent [18], and their interpretation more complex than inelastic X-ray scattering (IXS) from phonons.
} 
density fluctuation- we obtain for a mono-atomic system :

$$
\begin{aligned}
\frac{\partial^{2} \sigma}{\partial \Omega \partial E} & =\left[r_{0}^{2}\left(\hat{\epsilon}_{i} \cdot \hat{\epsilon}_{f}\right)^{2}\right]\left[\frac{k_{i}}{k_{f}} \sum_{I, F} P_{I}\left|\left\langle F\left|\sum_{k} f_{k}(Q) e^{i \vec{Q} \cdot \vec{R}_{k}}\right| I\right\rangle\right|^{2} \delta\left(E-E_{f}-E_{i}\right)\right] \\
& =\left[r_{0}^{2}\left(\hat{\epsilon}_{i} \cdot \hat{\epsilon}_{f}\right)^{2}\right]\left[\frac{k_{i}}{k_{f}} S(\vec{Q}, E)\right]
\end{aligned}
$$

where $\mathrm{k}$ runs only over the atoms while the individual electron contributions have been integrated into the radial part of the X-ray atomic form factor $f_{k}(\vec{Q})=-1 / e \int d \vec{r} e^{i \vec{Q} \cdot \vec{r}_{j}} \rho_{k}(\vec{r})$, which corresponds to the Fourier transform of the atomic charge density $\rho_{k}(\vec{r})$ for the $k$ th atom ${ }^{2}$.

Note that the term in the second square brackets of Eq. (2.2) is very similar to the coherent neutron scattering expression. In fact, replacing the X-ray atomic form factor with the (isotopic averaged) neutron scattering length $b_{k}$ for a given atom, one obtains the neutron coherent dynamical structure factor. Incoherent contributions to the inelastic X-ray scattering (IXS) cross section arise only from the Compton process, and are sizable only at higher energy transfer ( $>1 \mathrm{eV})$ [14].
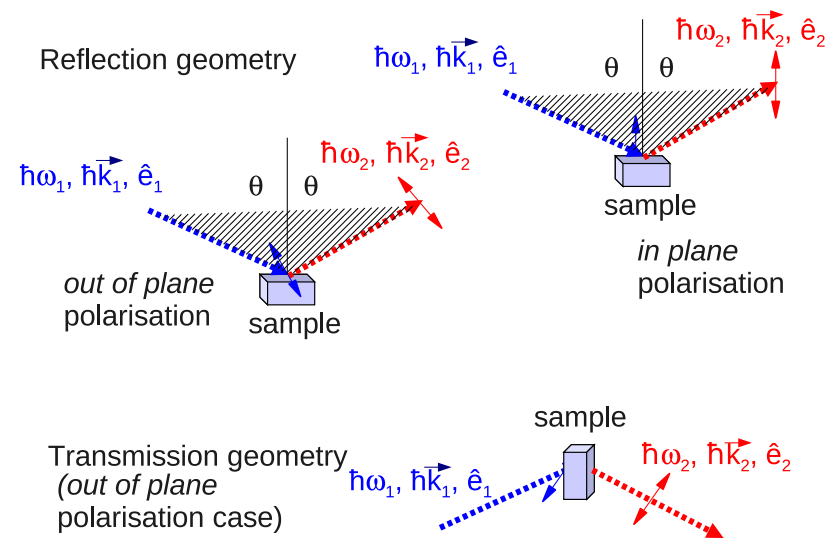

Figure 1. Light scattering geometry. The scattering angle $\theta_{s}$ between incoming and outcoming wave is twice the angle $\theta$.

Let's briefly analyse the different parts of the formula in detail, and compare it with the neutron case. As $r_{0}$ is $\sim 2.817 \mathrm{fm}$, the order of magnitude of the X-ray cross section is comparable to the neutron case, so we expect (and indeed observe) similar intensities for equivalent incoming beam intensities. The second term gives a contribution which depends on the light polarisation with respect to the plane of scattering. In the case of synchrotron radiation sources, the X-rays are linearly polarised in the orbit plane of the synchrotron.

If the scattering plane is the same as the synchrotron (Fig. 1, in-plane case), then the term $\left(\hat{\epsilon}_{i} \cdot \hat{\epsilon}_{f}\right)^{2}$ gives $\cos ^{2}\left(\theta_{s}\right)$, where $\theta_{s}=2 \theta$ is the scattering angle (see Fig. 1$)^{3}$. If the X-ray polarisation is perpendicular to it (Fig. 1, out-of-plane case), we have $\left(\hat{\epsilon}_{i} \cdot \hat{\epsilon}_{f}\right)^{2}=1^{4}$.

The second term, as we have stressed above, is very similar to the coherent neutron scattering term. The atomic form factor gives a contribution of the order $Z$ or less (the total charge of the atom) as $\max \left(f_{k}(Q)\right)=f_{k}(0)=Z$ for an isolated atom, and it is a decreasing function of $Q$. As a consequence, while the neutron scattering length is constant, $f(Q)$ depends on the norm of the momentum transfer

\footnotetext{
2 In the approximation of an isotropic atomic charge distribution.

3 At the ESRF this is the case for the ID28 spectrometer and the horizontal spectrometer of ID16.

4 ID16 vertical spectrometer.
} 


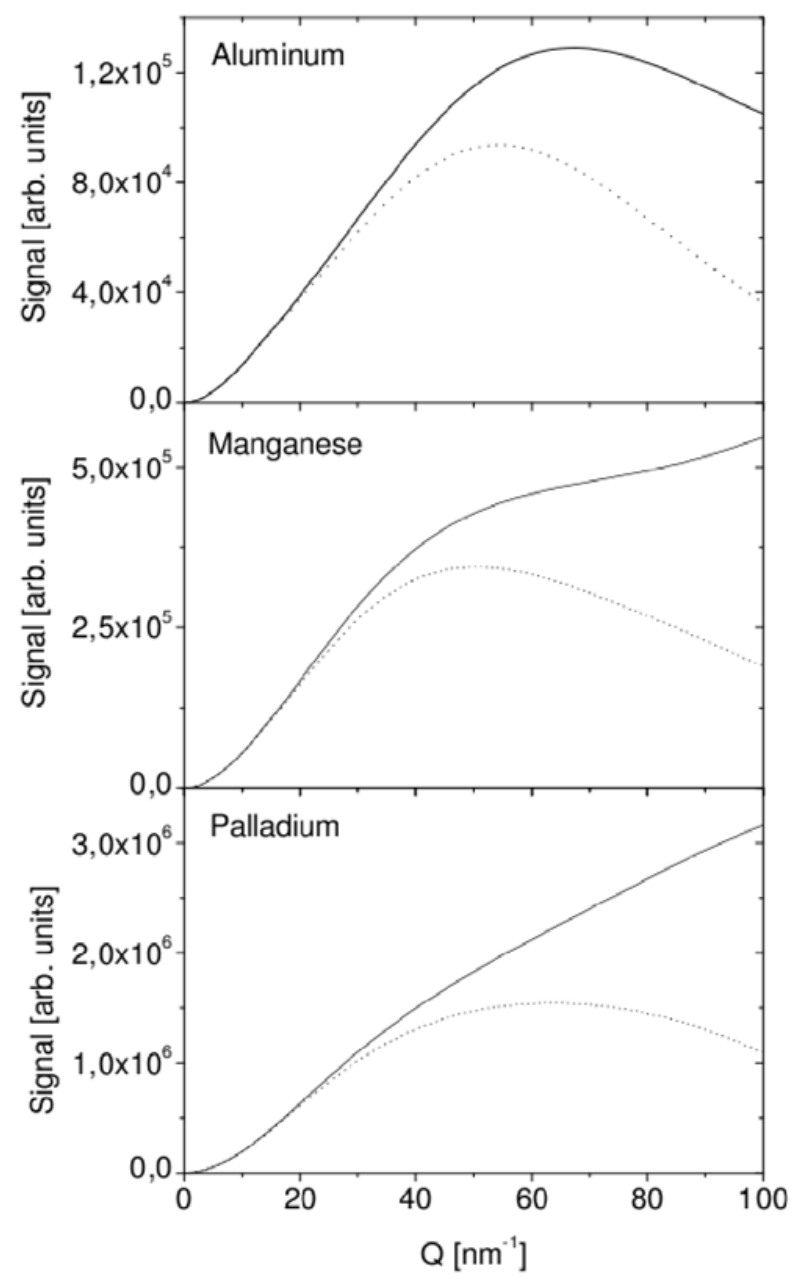

Figure 2. The product $(f(Q) \cdot Q)^{2}$ (solid line) and $(f(Q) \cdot Q)^{2} \cos ^{2}\left(\theta_{s}\right)$ (dashed line) as a function of $\mathrm{Q}$ for a photon energy of $22 \mathrm{keV}$ (from reference [15]).

for X-rays. This is due to the fact that the electronic cloud of an atom has a size comparable to the probe wavelength, while the nucleus has a much smaller size. Hence the nuclear density, as viewed by the strong interaction with the neutron, can be approximated by a delta function which gives a constant form factor in reciprocal space for thermal neutrons. In fact, this is almost the case for hard X-rays as well. Because the electronic density is concentrated within a very small radius for all but the valence electrons, $f(Q)$ decreases rather slowly with $Q$ for most of the elements. Note that, as Eq. (2.2) contains an expression for the dynamical structure factor similar to that of the coherent nuclear neutron scattering, it is also proportional to $\vec{Q} \cdot \hat{\mathrm{e}}_{k}^{n}(\vec{q})$ where $\hat{\mathrm{e}}_{k}^{n}$ is the polarisation of atom $k$ of the $n$th phonon mode at the reduced wavevector $\vec{q}$. It follows that the intensity of a mode as a function of the $Q$ wavevector norm is proportional (for a single atom) to $(f(Q) Q)^{2}$. We can see in Fig. 2 that the function $(f(Q) Q)^{2}$ is increasing up to very large momentum transfer with the exception of elements with very small $Z$ (like $\mathrm{H}$ and $\mathrm{He}$ atoms, for which the valence electrons are the only ones).

The total absorption cross-section for X-rays with energies above $10 \mathrm{keV}$ is limited in almost all cases $(Z>4)$ by the photoelectric absorption process. The photoelectric absorption, whose 


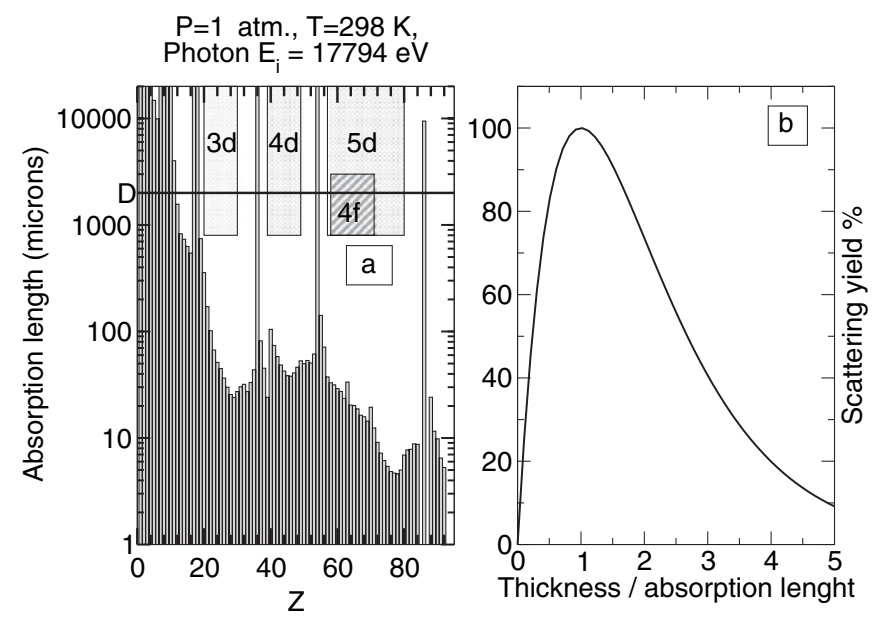

Figure 3. a. Absorption length for elements at ambient pressure and temperature conditions. Data from [21]. b. X-ray scattering yield in transmission.

cross-section $\sigma_{p e}$ is roughly proportional to $Z^{4}$, determines the optimal sample length along the scattering path. Consequently, the Thomson scattering channel is not very efficient for systems with high $Z$ despite of the $Z^{2}$ dependence of its cross-section $\sigma_{T S}$. Taking into account the absorption, the flux of scattered photons within an energy interval $\Delta E$ and within a solid angle $\Delta \Omega$ can be written as:

$$
N=N_{0} \frac{\partial^{2} \sigma}{\partial \Omega \partial E} \Delta \Omega \Delta E \rho L e^{-\mu L}
$$

where $N_{0}$ is the flux of the incident photons, $\rho$ is the number of scattering units per unit volume, $L$ is the sample length and $\mu$ is the total absorption coefficient. Fig. 3a shows the absorption length $1 / \mu$ for elements at ambient temperature and pressure, at an incident energy $E_{i}=17.794 \mathrm{keV}$, typical for high resolution IXS (see Tab. 1 below). The presence of large deviations from a smooth $Z^{4}$ dependence have their origins in either the strong increase of the atomic absorption when the incident energy $\mathrm{E}_{i}$ is close to that of an absorption edge or the large variation of densities for elements at ambient condition. In the same Fig. 3, panel b, the thickness dependence $L e^{-\mu L}$ of the scattering yield in the direction of the transmitted beam is reported. As we can see, the optimum thickness for a sample in transmission geometry (see Fig. 1) is $1 / \mu$. Therefore, the plot in Fig. 3, panel a gives an idea of the ideal thickness of elemental samples.

\subsection{Kinematics of X-ray inelastic scattering}

The light velocity in vacuum is $c=\lambda \nu$, where $\lambda$ and $v$ are the photon wavelength and frequency respectively and the dispersion law for light is simply:

$$
\hbar \omega=\hbar c k
$$

Then, writing the conservation law for the energy $E=\hbar \omega$ and momentum $\hbar Q$ exactly as in the neutron scattering case:

$$
\begin{gathered}
E=E_{i}-E_{f}=\hbar\left(\omega_{i}-\omega_{f}\right) \\
\hbar \vec{Q}=\hbar\left(\vec{k}_{i}-\vec{k}_{f}\right)
\end{gathered}
$$


Table 1. Most common reflections used for high resolution IXS in back-scattering set-up, and their wavelength, energy, monochromator resolution $\Delta E_{M}$ and overall spectrometer experimental resolution $\Delta E$ for ID28 [27].

\begin{tabular}{|c|c|c|c|c|}
\hline $\begin{array}{l}\text { Reflection } \\
\text { (h h h) }\end{array}$ & $\begin{array}{l}\text { Wavelength } \\
\text { (§) }\end{array}$ & $\begin{array}{c}\text { Energy } \\
(\mathrm{keV})\end{array}$ & $\begin{array}{c}\Delta E_{M} \\
(\mathrm{meV})\end{array}$ & $\begin{array}{c}\Delta E \\
(\mathrm{meV})\end{array}$ \\
\hline (7 77 ) & 0.8959 & 13.839 & 5.3 & $7.6 \pm .2$ \\
\hline$\left(\begin{array}{lll}8 & 8 & 8\end{array}\right)$ & 0.7839 & 15.816 & 4.4 & $5.5 \pm .2$ \\
\hline (999) & 0.6968 & 17.793 & 2.2 & $3.0 \pm .2$ \\
\hline$\left(\begin{array}{llll}1 & 11 & 11\end{array}\right)$ & 0.5701 & 21.747 & 1.02 & $1.5 \pm .1$ \\
\hline$\left(\begin{array}{lll}13 & 13 & 13\end{array}\right)$ & 0.4824 & 25.701 & 0.5 & $1.0 \pm .1$ \\
\hline
\end{tabular}

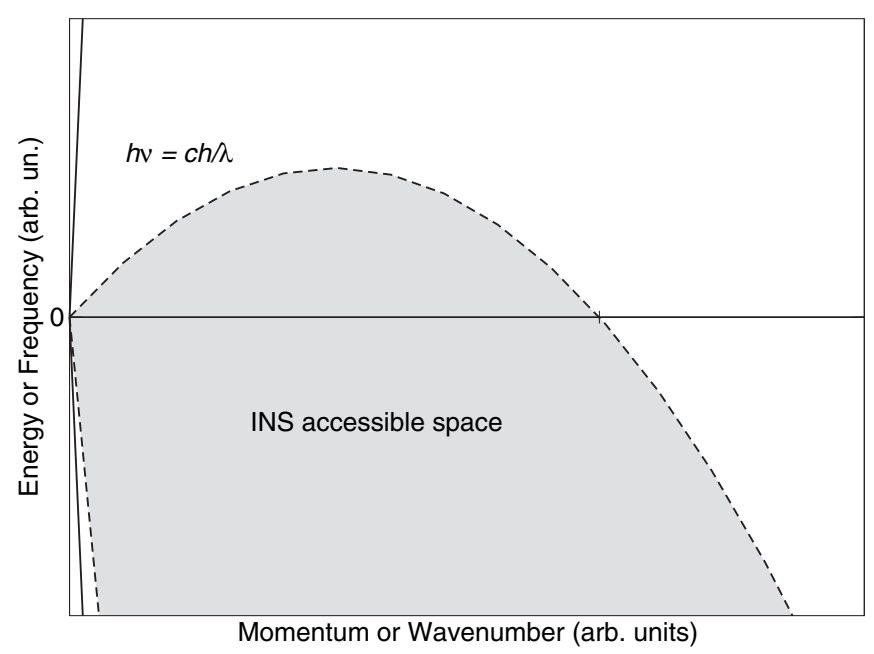

Figure 4. Kinematic limit for neutron and light inelastic scattering. For clarity, the slope are not to real scale, (the light dispersion would appear vertical).

allows to connect the probing wave (neutron or photon) energy shift $\hbar\left(\omega_{i}-\omega_{f}\right)$ and momentum change $\hbar\left(\vec{k}_{i}-\vec{k}_{f}\right)$ with the energy $\hbar \omega$ and momentum $\hbar \vec{Q}$ transferred to an excitation (phonon for instance) in the material under study.

The main difference with respect to INS lies in the dispersion law 2.4. Thermal neutrons have a quadratic dispersion with a slope never exceeding a few thousand $\mathrm{m} / \mathrm{s}$ which is well below the light velocity of $299,792,458 \mathrm{~m} / \mathrm{s}$, as shown in Fig. 4 . We will discuss the implications of this difference at the end of the lecture. We note here that this is a general result for light inelastic scattering and at any wavelength, i.e. applicable to visible light as well (Raman or Brillouin scattering).

A more subtle difference stands in the change of the momentum norm of the photon due to the inelastic process $\left(\hbar\left(\left|\vec{k}_{f}\right|-\left|\vec{k}_{i}\right|\right)\right)$ which is very small compared to the incident momentum norm $\hbar\left|\vec{k}_{i}\right|$, thus making the triangle formed by the three vectors $\vec{k}_{f}, \vec{k}_{i}$ and $\vec{Q}$ always isosceles, as shown in Fig. 5. This approximation is valid only well above the infrared regime, and applies only roughly to visible light (within 10\%), but very well to X-rays (with a precision of $10^{-7}-10^{-5}$ ), because the incident energy $(\sim 10-20 \mathrm{keV})$ is $4-5$ orders of magnitudes above the highest possible phonon mode $(\sim 0.1-0.2 \mathrm{eV})$. 

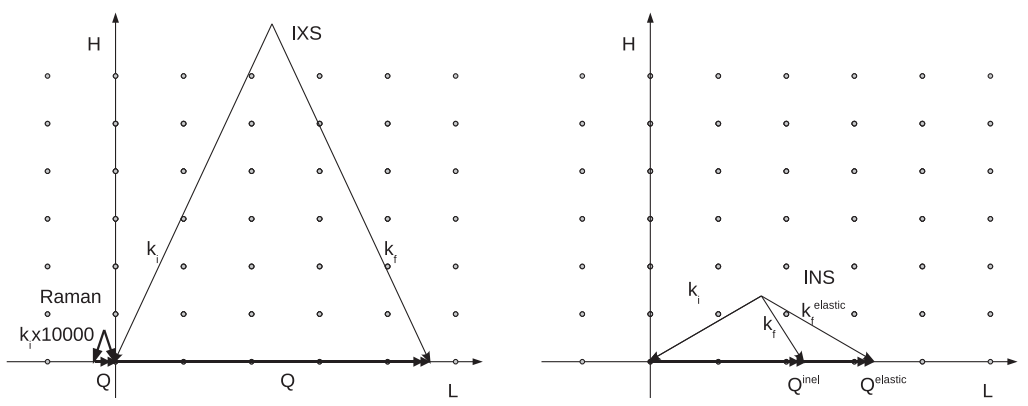

Figure 5. Kinematic condition for neutron and light inelastic scattering. Vectors are roughly to scale assuming visible light of $2.5 \mathrm{eV}$ energy, X-rays of $17.794 \mathrm{keV}$ and neutrons of $14 \mathrm{meV}$.

This fact has an important consequence, concerning the resolution. As the change in wavelength corresponding to the inelastic process is always very small compared to the initial value, the energy and $Q$ resolution are decoupled from the energy and wavevector transfer to a very good approximation. This means that the resolution can be considered constant throughout the whole $(\hbar \vec{Q}, \hbar \omega)$ momentum-energy space. Obviously, this greatly simplifies the data analysis of an IXS experiment.

A second important consequence is that any monochromator harmonics falls far outside the energy range of interest. As a consequence, there are no artifacts due to monochromator $\lambda / \mathrm{n}$ harmonics in the IXS phonon spectra.

The difference between X-ray and light scattering is that for the latter the wavelength is much larger than the characteristic distance between atoms or molecules so that the incident momentum $\hbar \vec{k}_{i}$ is very small compared to the typical zone boundary of the first Brillouin zone in the case of a crystal. Raman and Brillouin scattering experiments, therefore, access only a small portion of the reciprocal space around the first Brillouin zone center. In contrast, hard X-rays have a wavelength between 0.9 and $0.5 \AA$. Indeed, the IXS $Q$ radius is about $6-12 \AA^{-1}$, e.g. comparable to hot or epithermal neutrons thus allowing to explore a very large portion of the reciprocal space. This fact, together with the small variation of the photon momentum norm during the inelastic process, simplifies the practical realization of an IXS experiment: while for thermal neutron inelastic scattering experiments, one must always check to close the triangle in reciprocal space, composed of the incident wave-vector $\mathbf{k}_{i}$, the final one $\mathbf{k}_{f}$ and the scattering vector $\mathbf{Q}$, the triangle for IXS closes almost always. In fact, conditions which make the triangle impossible to close occur much more closer to $\Gamma\left(\sim 10^{-6} \AA^{-1}\right)$.

\section{HIGH RESOLUTION SPECTROMETER FOR X-RAY INELASTIC SCATTERING}

As already mentioned in the introduction (Sec. 1), the determination of phonon dispersions using Xray inelastic scattering requires a high energy resolution $\Delta E / E \sim 10^{-7}$, as photons of the appropriate wavelength $(\sim \AA)$ are in the $10^{4} \mathrm{eV}$ energy range. This is in fact relatively easy to achieve by high order Bragg reflections from a perfect crystal. The corresponding angular acceptance is however very small. If the source has a broad energy spectrum and/or a wide angular spread, most of the photons are rejected by the monochromator. The technical solution to overcome this requires a very bright source, i.e. at the same time intense, highly collimated and concentrated within a relatively small energy window. However, this is not sufficient even using state-of-the-art X-ray sources. It furthermore necessitates an optical configuration allowing to maximize the angular acceptance of perfect crystals. The first point has been achieved using third generation synchrotron sources with insertion devices called undulators, which we describe in Sec. 3.1. The second point has been achieved using a special configuration 

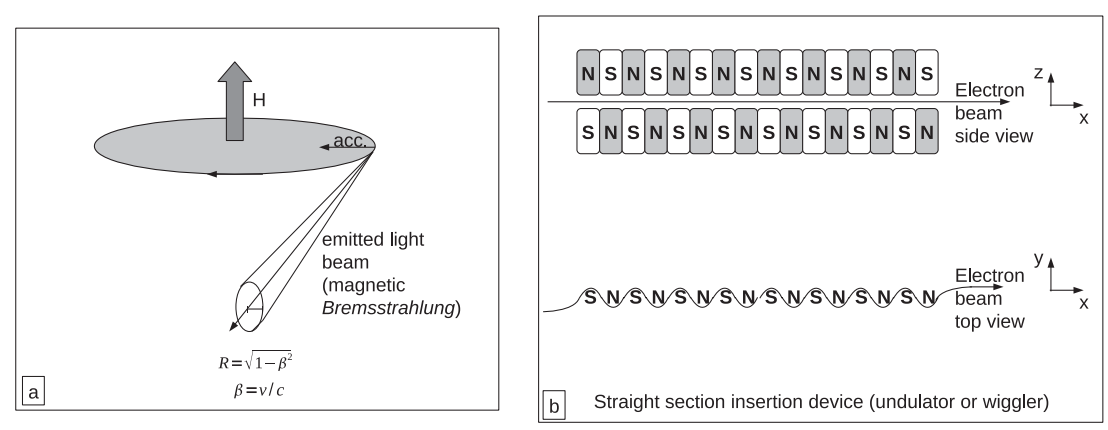

Figure 6. a. Synchrotron light emission from an uniform magnetic field $\mathrm{H}$, generating a circular orbit of electrons (positrons) with velocity v, close to the light velocity. b. Undulator insertion device. The deviations induced by the multipole are greatly exaggerated for clarity. (Adapted from [14].)

of the monochromator: the back-scattering configuration or the nested configuration. We are going to describe only the back-scattering configuration in 3.2. The nested configuration is described in Ref. [20,22,23].

\subsection{Light source requirements}

As explained previously, IXS requires an intense, highly collimated X-ray source with a relatively small energy band-width. Standard anode X-ray generators posses rather sharp emission lines, but they are superimposed to bremsstrahlung emission spread over a wide energy range. Furthermore, they have neither sufficient intensity, nor collimation, even in the rotating anode configuration, for the needs of high resolution IXS.

The emission from an accelerated charge, such as an electron or a positron, moving on a circular orbit, with a velocity close to the speed of light $\mathrm{c}$, is very intense and concentrated within a small solid angle $\phi=\sqrt{1-\beta^{2}}=\gamma^{-1}=m_{e} c^{2} / \epsilon_{e}$, where $m_{e}$ is the electron mass and $\epsilon_{e}$ its energy ${ }^{5}$. This emission is very close to that observed from a bending magnet in synchrotron radiation facilities (see Fig. 6 panel a). Although intense and relatively well collimated, the emission spectrum is that of bremsstrahlung i.e. widely spread in energy.

A further step is to put in line a series of magnet, with alternating direction of the field along its length, and create what is called an insertion device (ID, because inserted between two bending magnets), where the charges move on a "chicane" orbit, thus oscillating for an observer looking along the main axis of the ID, i.e. the direction $x$ in panel $\mathrm{b}$ of Fig. 6. In this configuration the emission from each single turn sums up either in intensity or in amplitude. The first case occurs when the oscillations give angular deviations from the ID axis larger than the emission angle $\gamma^{-1}$, and is called "wiggler". The second case, with amplitude summing up to give the total emission, is called "undulator". In that case, the emission is concentrated into a very small solid angle. Moreover, as the coherent addition of amplitudes is only valid at one particular wavelength (and its harmonics), the undulator emission is quasi-monochromatic [14], with emission lines only a few hundreds of $\mathrm{eV}$ wide. This source could have the tight requirements for high resolution inelastic X-ray scattering. We will see, in the next section 3.2, that to obtain a small band-with from a perfect crystal it is necessary to use very high order reflections. As a consequence, a relatively high energy is required $(\sim 15-25 \mathrm{keV})$. Insertion devices working in the undulator regime at these energies are possible only on high energy storage rings, limiting de facto

\footnotetext{
5 The detail of the angular distribution of a radiation emitted by an accelerated charge is described in general in Ref. [24], and its application to synchrotron radiation (magnetic bremsstrahlung) is described in detail in Ref. [15], Ch. 1; see also Ref. [14], Ch. 2. Here we limit the description to the minimum necessary to understand the overall picture.
} 

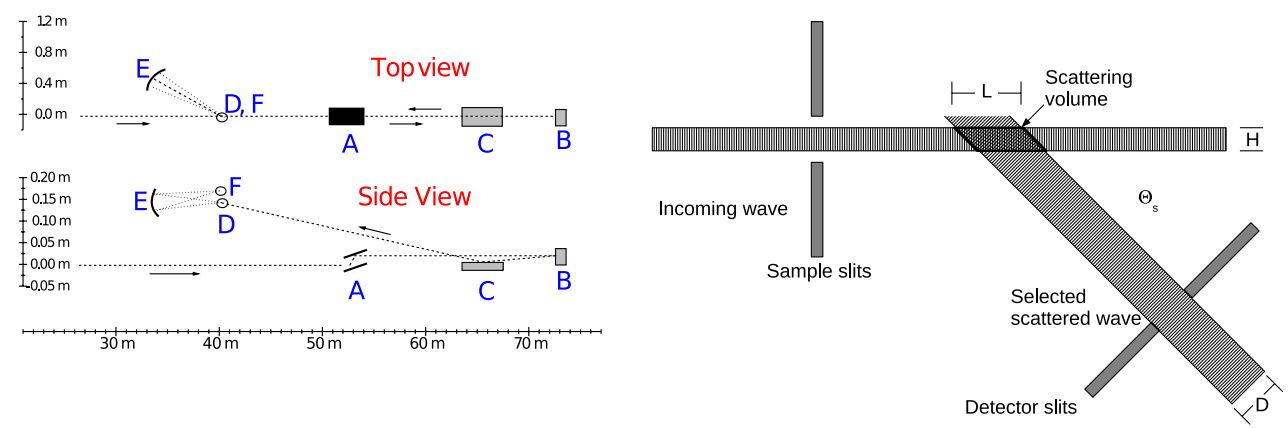

Figure 7. Left: Optical scheme of a back-scattering IXS spectrometer for phonon studies (ID16 and ID28 at the ESRF) [25]. A: High heat-load silicon (1 111 ) monochromator; B: High resolution back-scattering monochromator; C: toroidal mirror; D: sample; E: spherical crystal analyser; F: Detector. Note the very different length scales in the horizontal and vertical plane. See also Ref. $[13,20]$. Right: bird's view of the scattering volume defined by the incoming beam lateral size and detector slit projection L (see text).

the possibility to build this kind of experiments only to a few synchrotron facilities, namely : ESRF (France), APS (USA) and SPring-8 (Japan).

We note here a first important characteristics of high resolution IXS spectrometers : while neutron scattering sources have spherical (or $4 \pi$ ) emission, and collimation of the incoming beam is obtained through rejection of the neutrons not propagating within the accepted solid angle (either via collimators or guides), IXS starts with a very tightly collimated source, even before the monochromator. As an example, the very low beam divergence amounts to $0.0045 \times 0.0023^{\circ} \mathrm{Y} \times \mathrm{Z}$ on the ID16 and ID28 beam-line (ESRF, Grenoble).

\subsection{The back-scattering optical set-up}

The basic structure of the experimental set-up for high resolution inelastic X-ray scattering is the triple axis scheme, already discussed in the previous chapter by B. Hennion of this book. However, some specific arrangements are required in order to achieve high resolution with X-rays. We describe here in detail the back-scattering set-up, but most of the considerations are general. A full list of other possible set-ups are described in Ref. [20,23].

The three main components are the monochromator, the analyser crystals and the detector. We will describe the main monochromator and the analyser crystal components in detail, emphasising the technical solution chosen to achieve meV energy resolution in the following sections.

Let us start first with a general description of the beam-line optics, as sketched in Fig. 7. In front of the main monochromator one finds -in all the set-ups- a pre-monochromator (Fig. $7 \mathrm{~A}$ ). Its role is to reduce the still relatively large band-width of an undulator harmonic to a much smaller one, generally to about $1.5 \mathrm{eV}$. This is done in order to reduce the thermal power of the X-ray beam on the main monochromator, which otherwise would heat locally and create a thermal gradient. The resulting lattice parameter gradient would deteriorate the energy resolution of the main monochromator. The premonochromator usually consists of a double crystal, or a channel cut crystal, using the (1 111 ) Bragg reflection of silicon. It is usually cooled by liquid nitrogen. As the silicon thermal expansion has a minimum at around $100 \mathrm{~K}$, the thermal expansion coefficient is almost zero close to the Nitrogen boiling point while the thermal conductivity is still large (about $6 \mathrm{~W} /(\mathrm{cm} \mathrm{K})[26])$. The main monochromator (B) further reduces the band-width to the required $\mathrm{meV}$ range. It will be described in detail below (Sec. 3.3). Between the main mono and the sample, various configurations of mirrors (C) focus back the divergent beam onto the sample position (D). The detailed set-up can vary even for a single beam-line, according to the required spot size on the sample. For example, on the ID28 (ESRF, Grenoble) beamline, the toroidal 


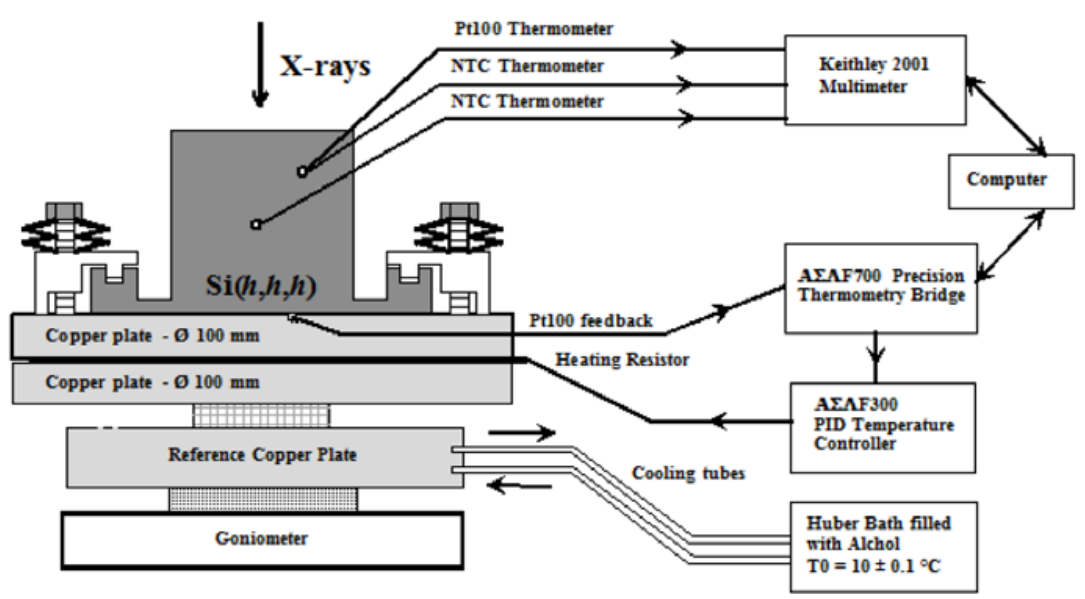

Figure 8. Details of the monochromator set-up [11,25].

mirror delivers a spot on the sample of $0.3 \times 0.09 \mathrm{~mm} \mathrm{H} \times \mathrm{V}$, while a combination of a cylindrical mirror focusing in the vertical plane (at the same position of the toroidal one) and a multilayer mirror focusing in the horizontal plane close to the sample (not shown) allows a focal spot of $0.03 \times 0.06 \mathrm{~mm}$ $\mathrm{H} \times \mathrm{V}$ on the sample.

The light scattered by the sample at a chosen scattering angle $\theta_{s}$ is first defined by the spectrometer slits, and then by analyser slits which control the solid angle seen by each analyser. This futher defines the spectrometer resolution in momentum space. The photons are then back-scattered by the analysers (E) on the detectors $(\mathrm{F})$, where the Bragg scattering angle for the energy analysis is defined by a second set of square slits (or pin-holes) in front of each detector, with $2 \mathrm{~mm}$ lateral size in the ID28 and ID16 set-up at ESRF. Finally, the photons are detected by a customised monolithic silicon detector in single photon counting mode.

The combination of incoming beam spot size on the sample (sample slits before the sample are only used to define the optical path) and detector and analyser slits define a sample volume, with size $\mathrm{H} \times \mathrm{V}$ in the plane perpendicular to the incoming beam as described above, and a length $L=\frac{D}{\sin \left(\theta_{s}\right)}$ where $\mathrm{D}=2 \mathrm{~mm}$ is the opening of the detector slits, as shown in Fig. 7, right panel. Note that $\mathrm{H}$, V are smaller than $\mathrm{D}$, which in turn is already a lower limit for L. This represents an upper limit for the scattering volume, as it is, in most cases, strongly limited by the sample absorption length $1 / \mu$ as shown in Fig. 3.

\subsection{The monochromator and the energy scale calibration}

The pre-monochromated X-ray beam with an energy resolution of typically $1.5 \mathrm{eV}$ must be further conditioned to a spectral bandwidth of few $\mathrm{meV}$, i.e. to a relative energy resolution of $\frac{\Delta E}{E} \sim 10^{-7}$ as explained in the introduction part of this lecture. We have seen that this condition is achieved using the Bragg reflection of a single crystal, i.e. similarly to what is done on the triple axis INS spectrometers (see the chapter by B. Hennion in this book for further details). However, several specificities are worth mentioning for IXS. First of all, the crystal is an almost perfect one with extremely small (virtually no) mosaic spread, very few defects and particular care is taken in order to avoid any mechanical or thermal stresses [11] (see Fig. 8).

This implies that the Bragg reflection profile is the one predicted by the dynamical theory of diffraction in reflection conditions $[14,15]$, which cannot be reduced to a simple mathematical function such as a Gaussian. We will see later and in detail this point (Sec. 3.4). Just let us underline here that the 
width of a Bragg reflection can be written as :

$$
\frac{\Delta E}{E}=\frac{d_{h k l}}{\pi \Lambda_{e x t}}
$$

where $d_{h k l}$ denotes the lattice spacing, associated with the (hkl) reflection order, and $\Lambda_{\text {ext }}$ the primary extinction length, a quantity deduced within the framework of the dynamical theory of X-ray diffraction. $\Lambda_{\text {ext }}$ increases with increasing reflection order. As a result the energy resolution increases when we select a higher order reflection, for a given fixed Bragg angle. This leads to the counter-intuitive result that for IXS the higher the photon energy the better the resolution. Table 1 summarises the most usual back-scattering reflections of type ( $\mathrm{H} \mathrm{H} \mathrm{H})$.

On a standard INS 3-axes set-up, the energy shift is selected using the scattering angle of either the monochromator or the analyser, according to Bragg's law. In a high resolution IXS set-up, this is done by acting on the pre-monochromator only to select the wavelength and -accordingly- the reflection order of the main monochromator, the latter being kept at a fixed angle in extreme back-scattering condition. The meV-resolution energy change is therefore not done by changing the scattering angle, but by varying the lattice spacing $d_{h k l}$. If one writes the Bragg law as :

$$
n \lambda=2 d_{h k l}(T, P) \sin \left(\theta_{S} / 2\right)
$$

one has the choice of either changing the scattering angle $\theta_{S}$ or the crystal plane $d_{h k l}$-spacing to vary $\lambda$. Varying the crystal plane $d_{h k l}$-spacing can be done by changing the $(\mathrm{P}, \mathrm{T})$ condition i.e. by varying either the temperature or the pressure ${ }^{6}$. Here we restrict ourselves to the most common case ${ }^{7}$ where only the temperature is varied. The lattice spacing parameter changes with the same law as the volume thermal expansion of the crystal :

$$
d_{h k l}(T)=d_{0}^{h k l}(1+\alpha(T) \Delta T)
$$

where $d_{0}^{h k l}$ is the lattice spacing at the reference temperature $T_{0}, \Delta T=T-T_{0}$. The volume expansion coefficient $\alpha(T)$ can be developed as :

$$
\alpha(T)=\alpha_{0}+\beta \Delta T+O\left(\Delta T^{2}\right)
$$

hence the Bragg law 3.2 can be developped as :

$$
n \lambda=2 d_{0}^{h k l}(1+\alpha(T) \Delta T) \sin \left(\theta_{S} / 2\right)
$$

Therefore, the difference between incoming and outcoming wavelength writes (for $\mathrm{n}=1$ ):

$$
\lambda-\lambda_{0}=\Delta \lambda=2 d_{0}^{h k l} \alpha(T) \Delta T \sin \left(\theta_{S} / 2\right) \approx 2 d_{0}^{h k l} \alpha_{0} \Delta T \sin \left(\theta_{S} / 2\right)=C \Delta T
$$

where $\lambda_{0}$ is the wavelength selected by one of the crystals kept at $T_{0}$ and $C=2 d_{0}^{h k l} \alpha_{0} \sin \left(\theta_{S} / 2\right)$ is a constant keeping the reflection order and scattering angle fixed. In contrast to typical X-ray monochromators which are often kept at liquid nitrogen temperature ${ }^{8}$ where the volume expansion coefficient is close to zero, here we need a sizable silicon volume expansion. The system is therefore kept around ambient temperature.

Using the photon dispersion law in vacuum Eq. (2.4) for a scattering angle of $\theta_{S} / 2=89.98^{\circ}$ (as on ID28 at ESRF, Grenoble) with the Silicon (9 9 9) Bragg reflection and using just the first order of the thermal expansion coefficient $\alpha_{0} \approx 2.58 \times 10^{-6} \mathrm{~K}^{-1}$ we obtain: $E(\mathrm{meV})=0.02195 / T\left(\mathrm{~K}^{-1}\right)$, i.e. a change of about $20 \mathrm{mK}$ of the monochromator temperature gives a change of about $1 \mathrm{meV}$ on the incident photons. Similar values are obtained for the other reflections. It is therefore necessary to have a thermal regulation in the $\mathrm{mK}$ range in order to minimize thermal instability effects on the

\footnotetext{
6 Or, more generally, by some kind of mechanical stress.

7 A pressure induced $d$-spacing change monochromator has been realised, e.g. at the ESRF, but its use is uncommon.

${ }^{8} \mathrm{As}$ it is the case here for the pre-monochromator, see above spectrometer description.
} 


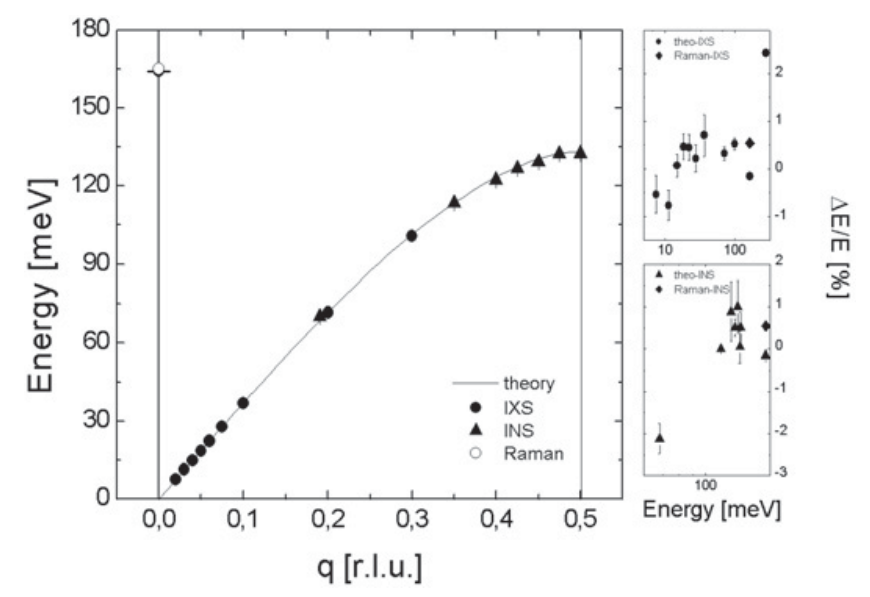

Figure 9. Comparison between experimental (IXS, INS and Raman scattering) and theoretical results for the phonon dispersion in diamond along the $\left(\begin{array}{lll}1 & 1 & 1\end{array}\right)$ direction from Ref. [25]. For the IXS data the temperature-energy conversion was performed as described in the text and Ref. [25]. Right: Relative deviation between IXS (top), INS (bottom) experiments and theory as a function of energy transfer on a logarithmic scale.

energy resolution and scale. This is achieved by a careful design of the thermal exchange between the monochromator (and analyser) and the thermal bath monitored by a commercial electronic thermal regulation system $[11,25]$. In the reality, it is not an absolute temperature which is considered but the temperature difference between the monochromator and the analyser. It is possible to precisely define the zero of the energy scale using the position of the maximum of intensity of any strong elastic diffuse scattering. Using the second order of the thermal expansion Eq. (3.4), one can precisely define the relative energy shift up to very large energy transfers with a precision better than $1 \%$ up to $170 \mathrm{meV}$ as shown in Fig. 9, and within 2\% for even larger energy transfers [25].

\subsection{The analysers and the instrumental resolution function}

The working principle of the analyser crystals is very similar to that we presented for the monochromator. However, the scattered X-rays from the sample spread in all directions which has the consequence of loosing the advantage of a highly collimated planar wave. It is therefore necessary to find a compromise between the very small angular acceptance of a perfect crystal Bragg reflection and the large angular spread of the scattered photons.

Let's take a cubic crystal of side $2 \mathrm{~d}$, with the diffraction plane ( $\mathrm{h} \mathrm{h} \mathrm{h}$ ) tangent to a sphere of radius $\mathrm{R}$ centred on the sample, as in Fig. 10, top panel. The above mentioned compromise, in back-scattering geometry, means to match the angle $\alpha=\arctan (d / R)$ (see Fig. 10, top panel) with the silicon $(h h h)$ high order Bragg reflection angular acceptance, which is, for the typical (11 11 11) case, of $\sim 0.0045^{\circ}$ (see Tab. 1) for the ESRF analysers. This results into a lateral size of about $1 \mathrm{~mm}$. A single analyser crystal of these dimensions, however, would gives an extremely high $Q$ resolution, useless in most cases for phonon excitation studies, and a very low spectrometer brilliance. The solution was to glue together many of such crystals $(\sim 12000)$ on a spherical support, with a radius of $6500 \mathrm{~mm}$, and a diameter of some $100 \mathrm{~mm}$, as displayed in the picture of Fig. 10, bottom, right. This corresponds to an angle close to $1^{\circ}$, both azimuthal and longitudinal. Slits in front of the analysers further reduce this solid angle to $0.2-0.6^{\circ}$. As this is the dominant contribution, the $Q$ resolution function is rather well approximated by a square line-shape.

The instrumental function in energy is composed of the convolution of the monochromator and analyser contributions. This results in a line-shape difficult to model theoretically, but constant 

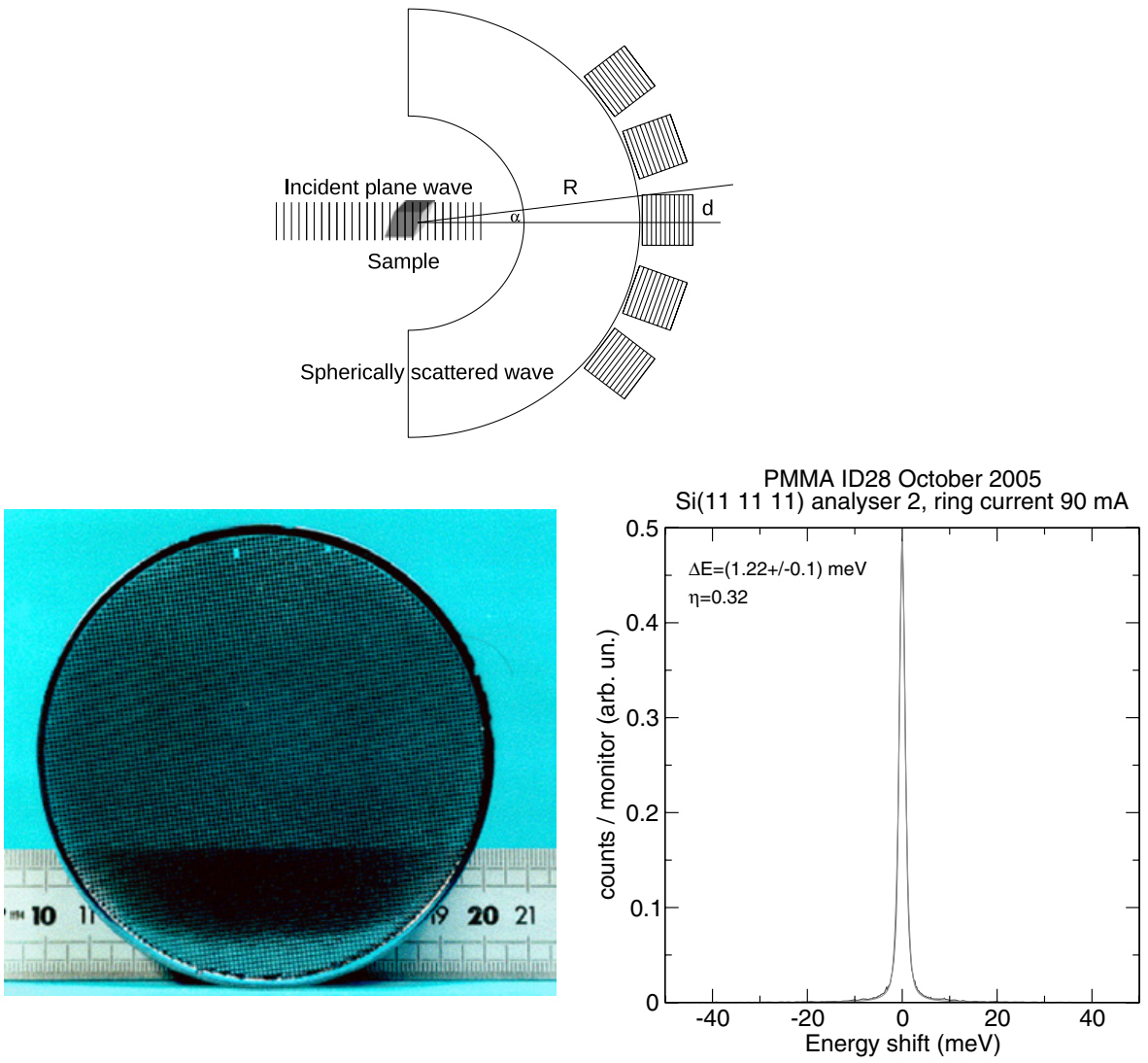

Figure 10. Top panel: schematic view of the analyser Bragg condition with respect to the spherically scattered wave; $d / R$ is greatly exaggerated for clarity. Left panel: Picture of a $6.5 \mathrm{~m}$ crystal analyser [25]. Right panel: IXS scan around zero energy transfer from a PMMA sample at $\mathrm{Q}=10 \mathrm{~nm}^{-1}$ and $\mathrm{T}=10 \mathrm{~K}[12,28]$. The experimental data were fitted to a Pseudo-Voigt function (solid line).

throughout the whole $(\hbar \vec{Q}, \hbar \omega)$ momentum-energy space as we mentioned previously in Sec. 2.2. It is usually measured using the elastic scattering from an amorphous sample having weak inelastic contributions. The measured energy resolution function is empirically modeled by a Pseudo-Voigt function :

$$
I(E)=I\left((1-\eta) \frac{(\Delta E / 2)^{2}}{\left(E-E_{0}\right)^{2}+(\Delta E / 2)^{2}}+\eta \exp \left(-\frac{(\ln 2)\left(E-E_{0}\right)^{2}}{(\Delta E / 2)^{2}}\right)\right)
$$

where $E=\hbar \omega=h v$ is the energy, $\Delta E$ is the full-width-half-maximum (FWHM) from which the experimental values of Tab. 1 (last column) are obtained. The instrumental line-shape parameter $\eta$ gives the relative weight of the Gaussian-type compared to the Lorentzian-type contribution, and it is usually close to 0.3 .

\section{COMPARISON WITH OTHER TECHNIQUES FOR THE MEASUREMENTS OF PHONON DISPERSION IN CRYSTALS}

For a comparison with other techniques, the first point is the kind of phenomena one wants to observe. We have already stressed (Sec. 2.1) that high resolution IXS can only probe dynamical fluctuations of 
density and not of magnetic moments or spins as with INS. This technique could be a useful complement to INS in special cases where it is necessary to disentangle phonon from magnetic excitations and when spin polarized INS is not available or difficult to interpret. The authors are, however, not aware of such application of combined IXS and INS to date.

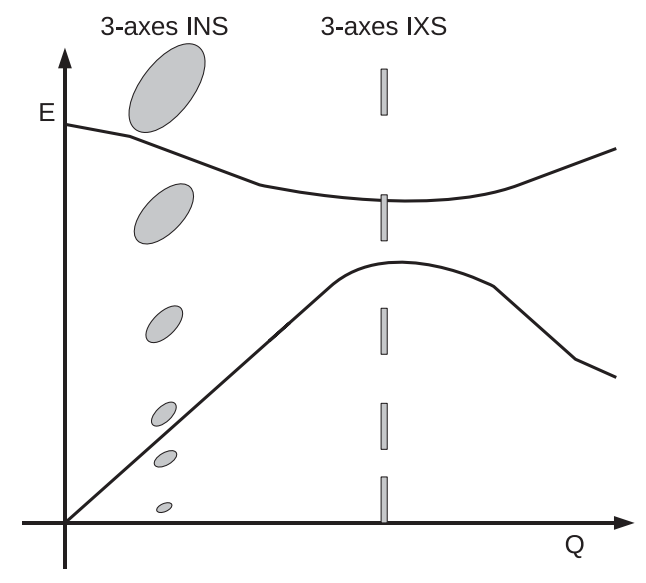

Figure 11. Schematic qualitative comparison of 3-axes thermal INS with IXS resolution function.

The fluctuations of density are seen by IXS through a modulation of the charge density. Phonons are one example, but other collective electronic excitations are possible. Indeed, as with Raman scattering, electronic excitations are visible using IXS [4,29]. However, the technique described here, which allows meV resolution, uses very hard X-rays, which are predominantly sensitive to the core electron density rather than to the valence electrons, as seen in Sec. 2.1. Therefore, high resolution IXS in the low energy region $(\sim 0-200 \mathrm{meV})$ is in general proportional to the atomic density fluctuations response function, which is quite close to the INS nuclear response ${ }^{9}$.

Furthermore, elements which possess isotopes strongly absorbing neutrons, such as cadmium, boron, lithium or mercury [30] or the elements with a very weak coherent scattering cross section such as vanadium, present no difficulties for IXS. We note that, as shown in Sec. 2.1, the order of magnitude of the IXS cross section is the classical electron radius $r_{0}$, which is of the same order of typical neutron scattering lengths. One would think that the $Z^{2}$ factor greatly enhances the scattering yields for high $Z$ elements but the IXS signal is limited by photoelectric absorption (see Fig. 3). Despite this constraint, it has been possible to measure elements of any $Z$, from hydrogen [31] to plutonium [32]. In fact, the very small attenuation length of many elements implies that very small thickness of such elements can be measured, as the absorption length corresponds to the optimal thickness in transmission (see Fig. 3). As the incident beam spot size on the sample is routinely in the $0.2 \mathrm{~mm}$ range and can be reduced to $0.03 \times 0.09 \mathrm{~mm} \mathrm{H} \times \mathrm{V}$, this allows probing very small sample volumes without significant loss of signal. This in turn permits one to study materials which are only available in small quantities $[32,33]$, or to perform experiments under extreme thermodynamic conditions such as very high pressure $[20,34]$. A problem of contrast arises when material are composed of both high and low $Z$ elements, for modes which have a mainly low $Z$ character ${ }^{10}$, as for the $\mathrm{Cu}-0$ bond-stretching mode in the cuprate case $[35,36]$. However the experiments can still be done if care is taken to use experimental conditions

\footnotetext{
${ }^{9}$ Nota bene: at high energies, away from the scattering by phonons, electronic excitations are visible as well. High energy resolution will require just higher integration $e . g$. in time or Q space.

${ }^{10}$ Meaning, their eigenvector is dominated by $u$ displacements of the lighter atoms.
} 
Table 2. Comparative summary of triple-axis ins and ixs characteristics. Short-cuts are made for clarity, more precise and detailed discussion can be found in the corresponding sections.

\begin{tabular}{||l|c|c|r||}
\hline & neutrons & X-rays & Section \\
\hline Phonons & yes & yes & 2.1 \\
Magnons & yes & no & 2.1 \\
Scattering & coherent + incoherent & coherent only & 2.1 \\
Coherent cross section*(barns) & $1-30$ & $10^{1}-10^{4}$ & 2.1 \\
Typical penetration depths & $\mathrm{cm}$ & $0.01-1 \mathrm{~mm}$ & 2.1 , see Fig. 3 \\
Spot size on the sample & $\mathrm{cm}$ & $30-300 \mu \mathrm{m}$ & 3.3 \\
Kinematic limitations & yes & no & 2.2 \\
$\lambda /$ n contaminations & yes & no & 2.2 \\
Energy resolution & $5-1 \%$ of $\mathrm{E}_{i}$ & $5.5-1.5 \mathrm{meV}$ & 3.3 , see Tab. 1 \\
Typical collimation & & & 3.3 \\
incident & $1^{\circ}$ & $0.0045^{\circ}$ & 3.4 \\
scattered & $1^{\circ}$ & $0.2^{\circ}$ & \\
\hline
\end{tabular}

*Natural isotope average for neutrons.

such as low temperature and large $Q$ transfer, which help at minimizing the contrast like it is explained in the above mentioned Ref. [35,36].

The cross section for X-rays has only a coherent contribution as there is no equivalent of isotopic disorder with X-rays. It is therefore observed that there is a very small or no elastic line away from the Bragg peaks in perfect crystals as can be seen e.g. in the energy spectrum of diamond (see Fig. 6 in Ref. [25]). Therefore, only static or quasi-static structural and/or chemical [35] disorder, contribute to the quasi-elastic signal.

Another important difference comes from the kinematics. As we have seen in Sec. 2.2 almost any energy transfer E can be reached at arbitrary momentum transfers Q. This was in fact the scientific case at the origin of the technique [10], as it allows one to determine dispersion with a sound velocity larger than that of the neutron even within the first Brillouin zone. This is important in liquids [10,13,30,31] and amorphous systems [37] as well defined excitations can be only be identified at small momentum transfers. This particular characteristics is obviously shared by all light scattering techniques, and indeed it is possible to measure e.g. the acoustic mode of water using Brillouin scattering. The large wavelength of visible light, however, limits the measurements to very small $Q$ values, and does not allow dispersion studies up to several tens of $\mathrm{nm}^{-1}$ in momentum transfers.

A further, important point, comparing INS to IXS, concerns the resolution. It is usually said that INS has a better energy resolution. In fact, the situation is more subtle, at least compared to the standard INS 3-axes technique. In that case, a more precise statement is that 3-axes INS has a resolution proportional to the incident energy, while IXS has a fixed one (see sketch in Fig. 11). This makes the INS 3-axes energy resolution up to an order of magnitude better for an energy range 0-50 meV. But at higher energy, up to about $100 \mathrm{meV}$, the two resolutions become roughly equivalent [36], and the IXS one is potentially even better at very high energy. Another important point concerning the resolution stands in the Lorentzlike tails of the IXS energy resolution (see Fig. 10 and Eq. (3.7)) making difficult to sort out weak modes close to strong ones or for samples with a strong elastic line [35]. This limitation is particularly drastic close to Bragg reflections [38]. It is however possible to measure very close to a strong elastic line thanks to the very high $Q$-resolution available. Indeed, while for the energy resolution the advantage lies on the INS side, for $Q$ resolution it is mostly the other way. For INS there is no theoretical limit for the $Q$ resolution which is set by the collimator choice, but the $4 \pi$ nature of the source makes it difficult to use a very tight collimation of the incident beam. Moreover, the large spot size and low scattering yield make necessary the use of large crystals, which often have a large mosaic spread. For IXS, the limitation on the source is reversed : only a very tight collimation of the beam is available (for the technical reason 
detailed in Sec. 3.2) making the angular divergence as small as $\sim 0.0045^{\circ}$, and the small spot size on the sample allows studying very high quality (small) crystals with extremely small mosaic spread $[36,38]$ or even a single grain in polycrystalline materials [39].

To complete the comparison with INS, we note that not only IXS spectra do not have any incoherent scattering, but also the multiple scattering is very low as it is in general strongly reduced by the high photoelectric absorption cross section. As a result, the IXS background is extremely low and flat, with the electronic noise of the detector as a main source, of about 1 counts per minute. This characteristic further simplifies the data analysis and also partially compensates for the difficulties due to the Lorentzian-type tails of the energy resolution function, for example when studying a strongly damped mode $[33,38]$. This strong reduction of multiple scattering processes together with total rejection of monochromator $\lambda / n$ harmonics, makes artifacts in IXS spectra very uncommon.

We already pointed out in this section and in Sec. 2.2 that Raman scattering is limited to the zone center (see Fig. 4). This difference can be seen as a complementarity to IXS for Raman active phonon modes. The $\mathrm{E}_{2 g}$ mode in $\mathrm{MgB}_{2}$ [38] is one good example. We note, however, that the cross section for visible photons explicitly includes an electron-phonon term [40], making the interpretation of Raman data more difficult [38] in some specific case while the IXS cross section is in general as simple as the coherent INS one (see Eq. (2.2)).

\section{Acknowledgements}

MD would like to acknowledge useful discussion with A. Shukla.

\section{References}

[1] P. Brüesch. In M. Cardona, P. Fulde, K. von Klitzing, and H.-J. Queisser, editors, Phonons: Theory and Experiments II, volume 65 of Solid-State Science, page 123. Springer-Verlag, Berlin, Heidelberg, New York, 1982.

[2] M. Born. Rep. Prog. Phys., 9:294, 1942.

[3] J. W. M. DuMond and H. A. Kirkpatrick. Rev. Sci. Inst., 1:88, 1930.

[4] J. W. M. DuMond. Rev. Mod. Phys., 5:1, 1933.

[5] H. A. Rowland. American Journal of Science, 3:87, 1883.

[6] H. A. Rowland. Philosophical Magazine, 5:197, 1883.

[7] B. Dorner and J. Peisl. Nucl. Instr. and Meth., 208:587, 1983.

[8] E. Burkel, J. Peisl, and B. Dorner. Europhys. Lett., 3:957, 1987.

[9] E. Burkel. Inelastic Scattering of X-rays with Very High Energy Resolution. Springer Verlag, Berlin, Heidelberg, New York, 1991.

[10] F. Sette, G. Ruocco, M. Krisch, U. Bergmann, C. Masciovecchio, V. Mazzacurati, G. Signorelli, and R. Verbeni. Phys. Rev. Lett., 75:850, 1995.

[11] R. Verbeni, F. Sette, M.H. Krisch, U. Bergmann, B. Gorges, C. Halcoussis, K. Martel, C. Masciovecchio, J.F. Ribois, G. Ruocco, , and H. Sinn. J. Synchrotron Radiation, 3:62, 1996.

[12] C. Masciovecchio, U. Bergmann, M. Krisch, G. Ruocco, F. Sette, and R. Verbeni. Nucl. Instrum. Meth. B, 111:181, 1996.

[13] F. Sette, G. Ruocco, M. Krisch, C. Masciovecchio, and R. Verbeni. Physica Scripta, T66:48, 1996.

[14] J. Als-Nielsen and D. McMorrow. Elements of modern X-ray physics. John Wiley \& Sons, New York, 2001.

[15] J. Baruchel, J.-L. Hodeau, M.S. Lehmann, J.-R. Regnard, and C. Schlenker, editors. Neutron and Synchrotron for Condensed Matter Studies, volume 1: Theory, Instruments and Methods of HERCULES. Les Editions de Physique, Springer-Verlag, Les Ulis, Berlin, Heidelberg, New York, 1993. 
[16] P. M. Platzman and N. Tzoar. Phys. Rev. B, 2:3556, 1970.

[17] F. de Bergevin and M. Brunel. Acta Crystallogr. A, 52:314, 1981.

[18] J. P. Hill, G. Blumberg, Young-June Kim, D. S. Ellis, S. Wakimoto, R. J. Birgeneau, Seiki Komiya, Yoichi Ando, B. Liang, R. L. Greene, D. Casa, and T. Gog. Physical Review Letters, 100:097001, 2008.

[19] N.-W. Ashcroft and N. D. Mermin. Physique des solides. EDP Sciences Editions, Les Ulis, 2003.

[20] M. Krisch and F. Sette. Inelastic X-ray scattering from phonons. In M. Cardona and R. Merlin, editors, Light Scattering in Solids IX, volume 108 of Topics in Applied Physics, chapter 5, page 317. Springer, Berlin, 2006.

[21] X-ray interactions with matter. http://henke.lbl.gov/optical_constants/.

[22] H. Sinn, E. E. Alp, A. Alatas, J. Barraza, G. Bortel, E. Burkel, D. Shu, W. Sturhahn, J. P. Sutter, T. S. Toellner, and J. Zhao. Nuclear Instruments and Methods in Physics Research A, 467-468:1545, 2001.

[23] E. Burkel. Rep. Prog. Phys., 63:171, 2000.

[24] J. D. Jackson. Classical Electrodynamics, chapter 14, page 662. Wiley, New York, 1975.

[25] R. Verbeni, M. d'Astuto, M. Krisch, M. Lorenzen, A. Mermet, G. Monaco, H. Requardt, and F. Sette. Review of Scientific Instruments, 79:083902, 2008.

[26] Y. S. Touloukian and E.H. Buyco, editors. Thermophysical Properties of Matter. IFI/Plenum Pub., New York, 1970.

[27] http://www.esrf.eu/UsersAndScience/Experiments/HRRS/ID28/Characteristics.

[28] C. Masciovecchio, U. Bergmann, M. Krisch, G. Ruocco, F. Sette, and R. Verbeni. Nucl. Instrum. Meth. B, 117:339, 1996.

[29] A. Kotani and S. Shin. Rev. Mod. Phys., 73:203, 2001.

[30] T. Scopigno, G. Ruocco, and F. Sette. Rev. Mod. Phys., 77:881, 2005.

[31] A. Cunsolo, D. Colognesi, M. Sampoli, R. Senesi, and R. Verbeni. J. Chem. Phys, 123:114509, 2005.

[32] J. Wong, M. Krisch, D. L. Farber, F. Occelli, A. J. Schwartz, T. C. Chiang, M. Wall, C. Boro, and R. Q. Xu. Science, 301:1078, 2003.

[33] A. Shukla, M. Calandra, M. d'Astuto, M. Lazzeri, F. Mauri, C. Bellin, M. Krisch, J. Karpinski, S. M. Kazakov, J. Jun, D. Daghero, and K. Parlinski. Phys. Rev. Lett., 90:095506, 2003.

[34] M. d'Astuto, M. Krisch, M. Lorenzen, A. Mermet, G. Monaco, H. Requardt, F. Sette, R. Verbeni, P. Loubeyre, R. Le Toullec, F. Occelli, G. Fiquet, J. Badro, and F. Guyot. High Pressure Res., 22:73, 2002.

[35] M. d'Astuto, P. K. Mang, P. Giura, A. Shukla, P. Ghigna, A. Mirone, M. Braden, M. Greven, M. Krisch, and F. Sette. Phys. Rev. Lett., 88:167002, 2002.

[36] M. d'Astuto, G. Dhalenne, J. Graf, M. Hoesch, P. Giura, M. Krisch, P. Berthet, A. Lanzara, and A. Shukla. Phys. Rev. B, 78:140511, 2008.

[37] F. Sette, M. Krisch, C. Masciovecchio, G. Ruocco, and G. Monaco. Science, 280:1550, 1998.

[38] M. d'Astuto, M. Calandra, S. Reich, A. Shukla, M. Lazzeri, F. Mauri, J. Karpinski, N. D. Zhigadlo, A. Bossak, and M. Krisch. Phys. Rev. B, 75:174508, 2007.

[39] M. Mohr, J. Maultzsch, E. Dobardžić, S. Reich, I. Milošević, M. Damnjanović, A. Bosak, M. Krisch, and C. Thomsen. Phys. Rev. B, 76:035439, 2007.

[40] M. Cardona. Resonance phenomena. In M. Cardona and G. Güntherodt, editors, Light Scattering in Solids II, volume 50 of Topics in Applied Physics, chapter 2, page 19. Springer, Berlin, 1982. 